\title{
Describing right ventricular function
}

\author{
Anton Vonk-Noordegraaf and Nico Westerhof
}

\begin{abstract}
In pulmonary hypertension and other conditions, such as acute respiratory distress syndrome and left heart failure, right ventricular dysfunction is a prognostic determinant. Therefore, functional parameters pertaining to the right heart alone, i.e. independent of its loading conditions, should be used to quantify right ventricular disease progression and the results of treatment. Two methods to quantitatively describe right ventricular function are reviewed, the pressure-volume relationship and the pump function graph; examples of each method are given. How to estimate wall stress and how to describe diastolic ventricular properties are also discussed.
\end{abstract}

n 1943, STARR et al. [1] showed that, in dogs,

following electrocautery ablation of the right ventricle free wall pulmonary venous pressure changed little, and the dogs survived. Based on this finding they concluded that the right ventricle functions only as a passive conduit, since there were "no increments of venous pressure". This study suggested to many researchers that the right ventricular pump may be neglected and this might explain why so little attention was paid to the right ventricle in the following decades. In recent years, however, this view has changed dramatically, mainly because it became clear that not only in pulmonary hypertension, but also in other conditions, like acute respiratory distress syndrome and left heart failure, right ventricular function is a prognostic determinant [2]. In addition, the importance of the right ventricle in determining maximal exercise capacity became clear [3]. Most of the studies investigating the right ventricle in different disease conditions and at exercise characterise the right ventricle by parameters obtained by imaging alone. Examples of parameters obtained from imaging are stroke volume (SV) as a measure of global cardiovascular performance, right ventricular ejection fraction, i.e. SV over end-diastolic volume, and tricuspid annular plane systolic excursion, as measures of right heart function [4-6]. More recent advances allow measurement of regional myocardial shortening by means of speckle echocardiography or strain analysis by magnetic resonance imaging (MRI) through tagging [7, 8] and calculation of wall stress to get more insight into (changes in) muscle properties per se. Although the advantage of such noninvasive approaches is clear, and helps to improve our insight into right ventricular function, their disadvantage is that they describe right ventricular function in relation to its preload and afterload, and thus, cannot be considered a quantification of right ventricular function per se.

Functional parameters that pertain to the right heart alone, and that are thus independent of its loading conditions, are important for several reasons. First, preload (filling) and afterload (arterial load) vary between patients. Secondly, since most pulmonary arterial hypertension (PAH)-specific treatments will affect either preload or afterload, or both, the possible impact of the treatment on the right ventricle requires characterisation in a load independent manner. For example, to predict right ventricular function after lung transplantation a load independent characterisation of right ventricular function is mandatory.

Therefore, this short review will focus on the principles of measuring right heart function independent of its load and how these principles can be applied in the clinic.

\section{CHARACTERISATION OF THE RIGHT HEART \\ Systole}

The pressure-volume relationship: figure 1 shows the so-called pressure-volume relationship of the right ventricle $[10,11]$. This relationship is

\section{AFFILIATIONS}

Dept of Pulmonology, Institute for Cardiovascular Research VU University Medical Center, VU University, Amsterdam, The Netherlands.

\section{CORRESPONDENCE}

A. Vonk-Noordegraaf Dept of Pulmonology VU University Medical Center De Boelelaan 1117 1081 HV Amsterdam The Netherlands

E-mail: a.vonk@vumc.n

Received:

Oct 092012

Accepted after revision: Dec 082012

First published online: Jan 112013 
obtained by changes in ventricular filling. The details of the relationship and how to obtain the relationship are given in the figure caption. The slope of the end-systolic pressure-volume relationship (systolic elastance: Ees) is a generally accepted loadindependent characterisation of the cardiac pump. Changes in filling pressure by vena cava occlusion are the best approach but are often not practical or desirable. Therefore, methods have been developed to obtain Ees from measurement of right ventricular pressure (catheterisation) and volume (MRI) of a single beat [1214], making it a practical method to obtain a load-independent functional characterisation of the right heart. The pressure-volume loop of the right ventricle is, in general, more of a trapezoidal shape than the more rectangular shape of the left ventricle, probably as a result of the very compliant pulmonary tree [15].

In the pressure-volume diagram (fig. 1) the line connecting the end-diastolic volume point with the end-systolic pressurevolume point can be drawn and its slope, Ea, gives the so-called arterial elastance. This term is confusing since $\mathrm{Ea}$ is not characterising the elasticity of the arterial system but is related to total pulmonary vascular resistance $(R)$, mean pulmonary artery pressure (mPAP) over cardiac output (CO), $R=\mathrm{mPAP} / \mathrm{CO}$.

End-systolic ventricular pressure $(P$ es $)$ is closely related to mean pulmonary artery pressure, and therefore the simplification that $\mathrm{Pes} \approx \mathrm{mPAP}[16]$ is sometimes assumed (see limitations), so that $\mathrm{Ea} \approx \mathrm{mPAP} / \mathrm{SV}=\mathrm{R} / \mathrm{T}$, where $\mathrm{T}$ is the heart period ( $\mathrm{R}-\mathrm{R}$ interval).

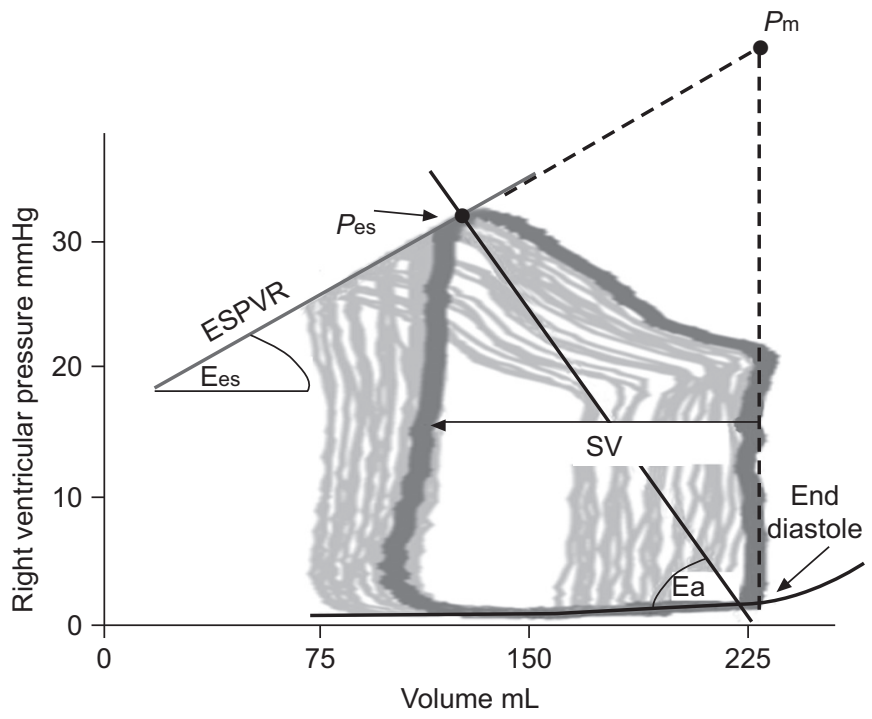

FIGURE 1. The pressure-volume relationship. A pressure-volume loop consists of four phases. Starting at end-diastole these phases are: isovolumic contraction, ejection, isovolumic relaxation and filling phase (black loop). The shallow diastolic phase shows large increases of volume with small increases in pressure. When diastolic filling is decreased by vena cava occlusion multiple loops are obtained (grey loops). The line connecting the end-systolic pressure-volume points is the end-systolic pressure-volume relationship (ESPVR). Its slope, Ees, is a measure of systolic ventricular function (contractility). The slope of the line connecting end-diastolic volume and end-systolic pressure, Ea, is called arterial elastance but is actually close to pulmonary vascular resistance over heart period: $R / T$. The ratio $\mathrm{Ees} / \mathrm{Ea}$ is a measure of ventriculo-arterial coupling. Maximal isovolumic pressure $(P \mathrm{~m})$ is determined with the single-beat method (see text), and with this method Ees $=(P \mathrm{~m}-\mathrm{Pes}) / \mathrm{SV}$. Pes: end systolic pressure; SV: stroke volume. Reproduced and modified from [9] with permission from the publisher.
The pressure-volume relationship is also used to estimate ventriculo-arterial coupling. It has been shown that when the ratio $\mathrm{Ea} / \mathrm{Ees}=1$ external work is maximised, while for $\mathrm{Ea} /$ Ees $=0.5$ cardiac efficiency is maximal [17]. In this context it should be mentioned that ejection fraction $(\mathrm{EF})$, rather than being a characterisation of the ventricle, is a coupling parameter. It turns out that EF is more closely related to Ea/Ees and to Ea [11].

Applying an even further simplification, SANZ et al. [18] calculated Ees by assuming that the intercept with the volume axis is negligible, thus giving Ees=Pes/ESV, where ESV is the end systolic volume. This assumption, however, underestimates Ees in a contractility dependent manner [14] and leads to $\mathrm{Ea} /$ Ees=1/EF-1 [11]. The pump function graph: figure 2 shows the so-called pump function graph that relates mean ventricular pressure and SV (for details see caption) [19]. This graph is obtained by changes in cardiac load. Again in practice this may not be possible or desirable, but the same single-beat methods $[12,13]$ can be used to find the (mean value of) maximal pressure to obtain a second point on the graph. The two points can then be fitted by a parabola [20]. The slope of the pump function graph is related to Ees. From the pump function graph maximal efficiency (close to optimal power output of the ventricle), is found when mean pressure is $66 \%$ of maximal pressure or $\mathrm{SV}$ is $58 \%$ of maximal SV (intercept of horizontal axis) [21, 22].

The relationship between the end-systolic pressure-volume relationship and the pump function graph have been discussed previously [11].

\section{Diastole}

The diastolic pressure-volume relationship determines filling and thus cardiac output (Frank-Starling mechanism). The

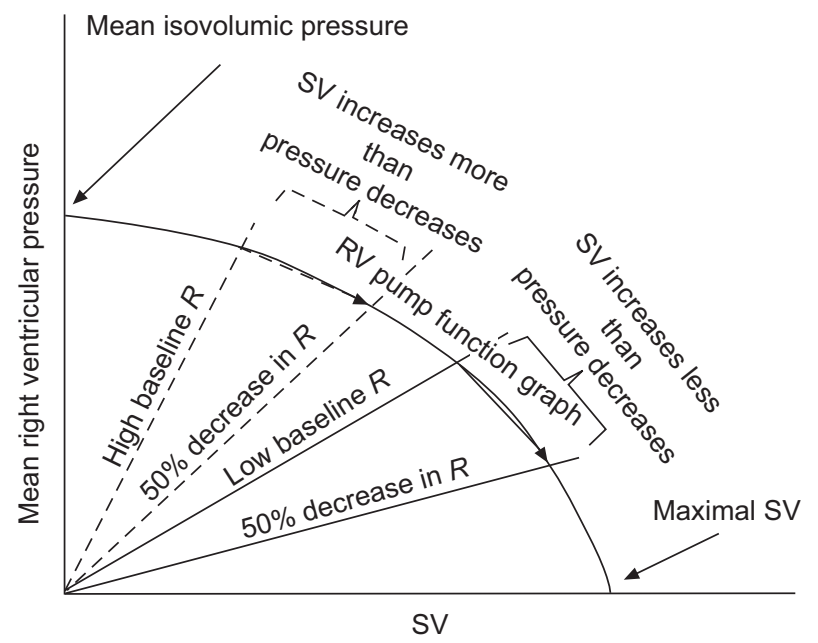

FIGURE 2. The pump function graph relates mean ventricular pressure and stroke volume (SV) and can be obtained by changes in arterial load. The slopes of the straight lines, are pulmonary vascular resistance, indicated by $R$, and are a measure of pulmonary arterial load and are proportional to Ea $\times \mathrm{T}$ (where $\mathrm{T}=$ heart period). The (mean) isovolumic pressure can be obtained by means of the singlebeat method. The relationship explains the effect of decreasing the arterial load on pressure and SV (and thus also on flow). When baseline $R$ is high, a decrease in $R$ mainly causes an increase in SV, while at lower baseline $R$ pressure is more affected. Ea: arterial elastance. 
pressure-volume inherently contains the information, but again, changes in filling are not practical. A straight line approach as a description of the diastolic pressure-volume relationship is not sufficiently accurate. Several descriptions of the curved diastolic pressure-volume relationship have been proposed. The best relationship is $\left.P=P=P_{\mathrm{o}}\left(\mathrm{e}^{\beta\left(V-V_{\mathrm{d}}\right.}\right)-1\right)$, with $V \mathrm{~d}$ the volume at negligible (transmural) pressure and $P_{\mathrm{o}}$ and $\beta$ the amplitude and curvature, respectively. Approximations have been used, such as $P=P_{0} e^{\beta V}$, a "asymptotic model" [23] and even simpler $P=P_{o} e^{\beta V}$ [24]. The third formula has only two parameters and can be used with, in principle, two data points. A single beat approach requires more simplifying assumptions, and is not yet available.

\section{Wall stress}

When information on the cardiac muscle is desired (segmental) wall shortening and wall stress are required. Wall shortening can be quantitatively obtained by echocardiography or cardiac MRI. Wall stress is not directly measurable and needs quantitative information on ventricular shape and size (e.g. radius of curvature (r) and wall thickness (h)). Laplace's law, $\mathrm{T}=\operatorname{Pr} / \mathrm{h}$, with $\mathrm{T}$ stress, and $\mathrm{P}$ (transmural) pressure allows for a rough estimate of wall stress [10]. The right ventricular radius varies greatly with location which makes accurate calculations doubtful. No generally accepted calculation of wall stress exists. At present a simple, easy to calculate estimate of wall stress is $\mathrm{T}=\left(1+3 V_{\text {lumen }} / V_{\text {wall }}\right) \mathrm{P}$ [25].

\section{Direct ventricular interaction}

Direct ventricular interdependency mediated via the interventricular septum plays an important role in right ventricular pressure overload. Therefore a complete description of cardiac function should take ventricular interdependency into account. However, it was shown that leftward septal bowing [26] is a consequence of prolonged right ventricular contraction, and occurs after pulmonary valve closure [27] and it is, therefore, unlikely that ventricular interdependence affects the description of right ventricular systolic function as described above.

\section{POSSIBLE LIMITATIONS OF THE PROPOSED METHODS}

Although the above described methods are, at present, the most optimal descriptions of right ventricular function they have limitations, which need further attention.

The slope of the isochrone with maximal slope, is designated as Emax, and may not be equal to the slope of the end-systolic pressure-volume relationship, Ees, which is obtained by multiple beats [28]. The "most left-top corners" of the pressure-volume loops are to be used to obtain Ees. As surrogate for $P$ es, mean arterial pressure [27] can be used, and since the dicrotic notch in arterial pressure and mean arterial pressure are closely related as well [16] this pressure can be used [29]. However, in practice mean pulmonary artery pressure is the most simple to obtain and suffers little from catheter artefacts. Nevertheless, future studies should clarify these issues.

All end-systolic pressure-volume relationships are nonlinear [30] but assumed to be linear, which is a workable approximation of reality [31-33].
To calculate Ea, the $P$ es and SV can be used, and $\mathrm{Ea}=P$ es $/ \mathrm{SV}$ [12]. It has been suggested that subtraction of left atrial (or pulmonary capillary wedge pressure) gives a better estimate of $R=\mathrm{Ea} \times \mathrm{T}$ [34]. However, it has not been shown which estimate of Ea is best for estimation of coupling (i.e. Ees/Ea).

In the calculation of Ees/Ea pressure drops out and the effects of the choice of end-systolic pressure become rather unimportant.

Systolic and diastolic ventricular function are both required for a full description of the right ventricle. Single beat methods are surrogates of the methods using changes in load: the better multiple beat methods. However, although the multiple beat method has shown its value in animal-related research, the risks of changing loading conditions in pulmonary hypertension patients are too high to use this technique in the pulmonary hypertension clinic.

End-systolic elastance is only a little different from maximal elastance [35], so that end-systolic elastance, using $P$ es, may be used in practice. Using peak (maximal) ventricular pressure as surrogate for $P$ es is not advisable, since it leads to errors. The end-systolic pressure-volume relationship is not straight, but in the working range a linear relationship seems acceptable. However, in extreme cases linearity may be lost [11].

In the single beat method, the assessment of isovolumic pressure and its maximal value in pressure-volume analysis, and mean value in the pump function graph approach, is based on the isovolumic part of the measured right ventricle pressure curve only and the fit is thus sensitive to errors and requires high quality right ventricular pressure curves. Using catheter-tip manometers and measuring more pressure curves can partially overcome this problem. The choice of the fitted curve, i.e. a sine wave [12-14], may also be a limitation and a "standard" isovolumic pressure curve obtained from measurements needs to be worked out [35].

The given methods describe the right ventricle in isolation. Since the right ventricle interacts on the left ventricle and vice versa, the question remains to be answered whether an optimal description also should incorporate ventricular direct and/or series interdependency [36, 37]. Ventricular interdependency in pulmonary hypertension needs further studies, but accounting for this effect will increase complexity, which may limit clinical usage.

The end-systolic pressure-volume relationship and the pump function graph depend on muscle contractility and ventricular wall mass. They characterise the ventricle as a whole but to derive muscle function other analyses should be carried out.

\section{EXAMPLES}

An example of the use of the pressure-volume relationship is given in figure 3 [9]. It may be seen that Ees is much higher in experimental pulmonary hypertension than in the control. The increase in ventricular wall thickness, and possibly also in muscle contractility causes an increase in Ees. The pressurevolume relationships also give information on diastolic ventricular properties $(\mathrm{Ed})$ and it may be seen that $\mathrm{Ed}$ is also increased in pulmonary hypertension.

An example of the use of the pump function graph is shown in figure 4 [38]. The graphs are obtained through the single-beat 


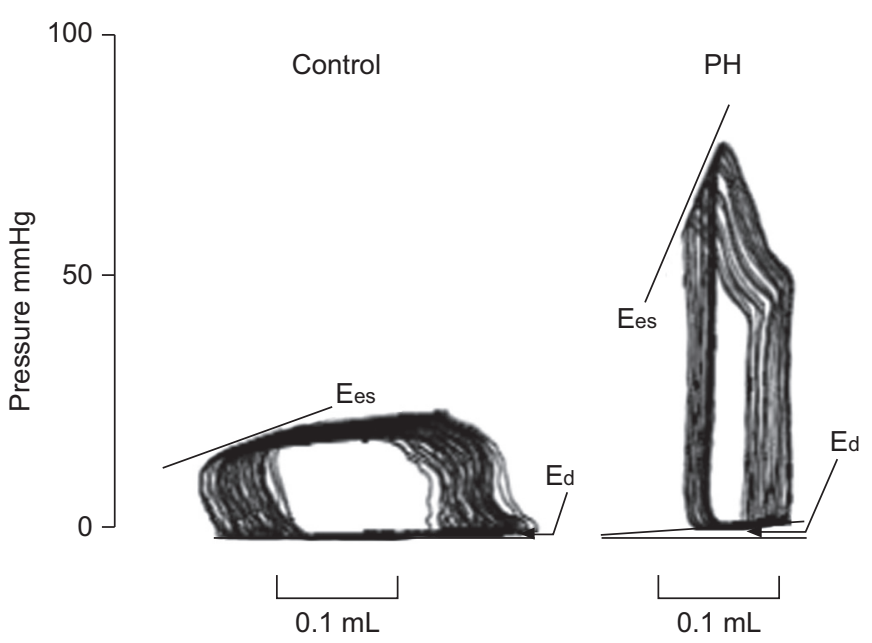

Relative volume

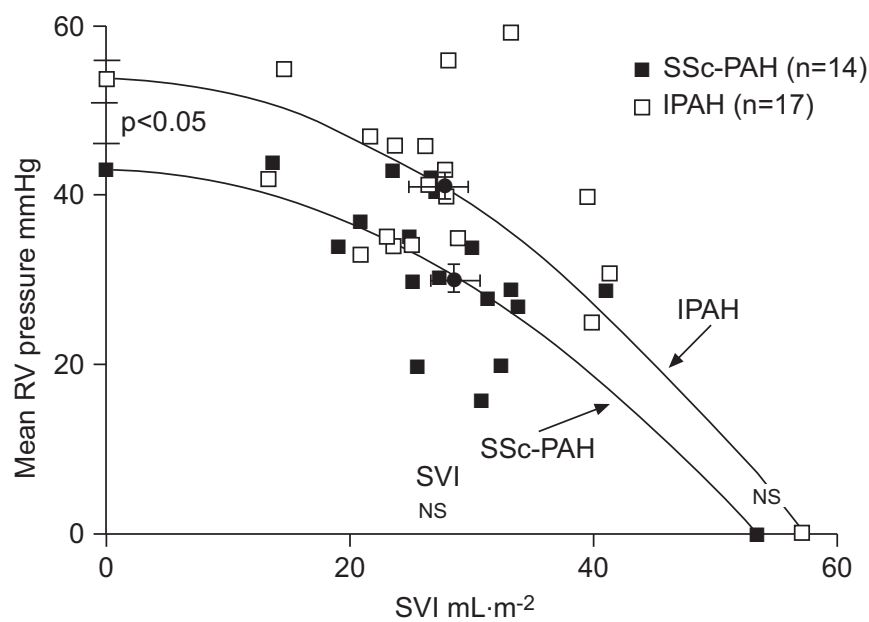

FIGURE 4. The pump function graph of patients with idiopathic pulmonary arterial hypertension (IPAH) and patients with systemic sclerosis associated pulmonary arterial hypertension (SSc-PAH). Stroke volume index (SVI) and maximal SVI are not different but isovolumic right ventricular pressure is higher in IPAH. The results are based on the single-beat method (see text). Reproduced and modified from [38] with permission.

of PAH specific medication and medication aimed to target the right ventricle can be addressed in the near future. In addition our insight into the mechanisms of right ventricular failure might improve if we combine these measurements with other meaningful parameters, such as parameters of myocardial perfusion or metabolism.

\section{STATEMENT OF INTEREST}

Conflict of interest information can be found alongside the online version of this article at www.erj.ersjournals.com

\section{REFERENCES}

1 Starr I, Jeffers WA, Meade RH. The absence of conspicuous increments of venous pressure after severe damage to the RV of the dog, with discussion of the relation between clinical congestive heart failure and heart disease. Am Heart J 1943; 26: 291-301.

2 Haddad F, Doyle R, Murphy DJ, et al. Right ventricular function in cardiovascular disease, part II: pathophysiology, clinical importance, and management of right ventricular failure. Circulation 2008; 117: 1717-1731.

3 La Gerche A, Gewillig M. What limits cardiac performance during exercise in normal subjects and in healthy fontan patients? Int $J$ Pediatr 2010; 2010: 791291.

4 van Wolferen SA, van de Veerdonk MC, Mauritz GJ, et al. Clinically significant change in stroke volume in pulmonary hypertension. Chest 2011; 139: 1003-1009.

5 van de Veerdonk MC, Kind T, Marcus JT, et al. Progressive right ventricular dysfunction in patients with pulmonary arterial hypertension responding to therapy. J Am Coll Cardiol 2011; 58 2511-2519.

6 Kind T, Marcus JT, Westerhof N, et al. Longitudinal and transverse movements of the right ventricle: both are important in pulmonary arterial hypertension. Chest 2011; 140: 556-557.

7 Mauritz GJ, Kind T, Marcus JT, et al. Progressive changes in right ventricular geometric shortening and long-term survival in pulmonary arterial hypertension. Chest 2012; 141: 935-943.

8 Marcus JT, Mauritz GJ, Kind T, et al. Interventricular mechanical dyssynchrony in pulmonary arterial hypertension: early or 
delayed strain in the right ventricular free wall? Am J Cardiol 2009; 103: 894-895.

9 de Man FS, Handoko ML, van Ballegoij JJ, et al. Bisoprolol delays progression towards right heart failure in experimental pulmonary hypertension. Circ Heart Fail 2012; 5: 97-105.

10 Suga H, Sagawa K, Shoukas AA. Load independence of the instantaneous pressure-volume ratio of the canine left ventricle and effects of epinephrine and heart rate on the ratio. Circ Res 1973; 32: 314-322.

11 Westerhof N, Stergiopulos N, Noble MIM. Snapshots of Hemodynamics: An Aid for Clinical Research and Graduate Education. 2nd Edn. New York, Springer, 2010; pp. 77-87.

12 Sunagawa K, Yamada A, Senda Y, et al. Estimation of the hydromotive source pressure from ejecting beats of the left ventricle. IEEE Trans Biomed Eng 1980; 27: 299-305.

13 Brimioulle S, Wauthy P, Ewalenko P, et al. Single-beat estimation of right ventricular end-systolic pressure-volume relationship. Am J Physiol Heart Circ Physiol 2003; 284: H1625-H1630.

14 Trip P, Kind T, van de Veerdonk MC, et al. Accurate assessment of load-independent right ventricular systolic function in patients with pulmonary hypertension. J Heart Lung Transplant 2013; 32: 50-55.

15 Friedman BJ, Lozner EC, Curfman GD, et al. Characterization of the human right ventricular pressure-volume relation: effect of dobutamine and right coronary artery stenosis. J Am Coll Cardiol 1984; 4: 999-1005.

16 Chemla D, Hébert JL, Coirault C, et al. Matching dicrotic notch and mean pulmonary artery pressures: implications for effective arterial elastance. Am J Physiol 1996; 271: H1287-H1295.

17 Sunagawa K, Maughan WL, Sagawa K. Optimal arterial resistance for the maximal stroke work studied in the isolated canine left ventricle. Circ Res 1985; 56: 586-595.

18 Sanz J, García-Alvarez A, Fernández-Friera L, et al. Right ventriculo-arterial coupling in pulmonary hypertension: a magnetic resonance study. Heart 2012; 98: 238-243.

19 Elzinga G, Westerhof N. Pressure and flow generated by the left ventricle against different impedances. Circ Res 1973; 32: 178-186.

20 van den Horn GJ, Westerhof N, Elzinga G. Interaction of heart and arterial system. Ann Biomed Eng 1984; 12: 151-162.

21 van den Horn GJ, Westerhof N, Elzinga G. Optimal power generation by the left ventricle. A study in the anesthetized open thorax cat. Circ Res 1985; 56: 252-261.

22 Toorop GP, Van den Horn GJ, Elzinga G, et al. Matching between feline left ventricle and arterial load: optimal external power or efficiency. Am J Physiol 1988; 254: H279-H285.

23 Noble MI, Milne EN, Goerke RJ, et al. Left ventricular filling and diastolic pressure-volume relations in the conscious dog. Circ Res 1969; 24: 269-283.

24 Gaasch WH, Cole JS, Quinones MA, et al. Dynamic determinants of left ventricular diastolic pressure-volume relations in man. Circulation 1975; 51: 317-323.

25 Arts T, Bovendeerd PH, Prinzen FW, et al. Relation between left ventricular cavity pressure and volume and systolic fiber stress and strain in the wall. Biophys J 1991; 59: 93-102.
26 Marcus JT, Gan CT, Zwanenburg JJ, et al. Interventricular mechanical asynchrony in pulmonary arterial hypertension: leftto-right delay in peak shortening is related to right ventricular overload and left ventricular underfilling. J Am Coll Cardiol 2008; 51: 750-757.

27 Mauritz GJ, Marcus JT, Westerhof N, et al. Prolonged right ventricular post-systolic isovolumic period in pulmonary arterial hypertension is not a reflection of diastolic dysfunction. Heart 2011; 97: 473-478.

28 Sagawa K. The end-systolic pressure-volume relation of the ventricle: definition, modifications and clinical use. Circulation 1981; 63: 1223-1227.

29 Konstam MA, Cohen SR, Salem DN, et al. Comparison of left and right ventricular end-systolic pressure-volume relations in congestive heart failure. J Am Coll Cardiol 1985; 5: 1326-1334.

30 Claessens TE, Georgakopoulos D, Afanasyeva M, et al. Nonlinear isochrones in murine left ventricular pressure-volume loops: how well does the time-varying elastance concept hold? Am J Physiol Heart Circ Physiol 2006; 290: H1474-H1483.

31 Dell'Italia LJ, Santamore WP. Can indices of left ventricular function be applied to the right ventricle? Prog Cardiovasc Dis 1998; 40: 309-324.

32 Kass DA, Beyar R, Lankford E, et al. Influence of contractile state on curvilinearity of in situ end-systolic pressure-volume relations. Circulation 1989; 79: 167-178.

33 Lankhaar JW, Rövekamp FA, Steendijk P, et al. Modeling the instantaneous pressure-volume relation of the left ventricle: a comparison of six models. Ann Biomed Eng 2009; 37: 1710-1726.

34 Morimont $\mathrm{P}$, Lambermont $\mathrm{B}$, Ghuysen $\mathrm{A}$, et al. Effective arterial elastance as an index of pulmonary vascular load. Am J Physiol Heart Circ Physiol 2008; 294: H2736-H2742.

35 Kind $\mathrm{T}$. Towards a better description of cardiovascular function in pulmonary hypertension. PhD thesis. VU University, Amsterdam, The Netherlands, 2011.

36 Belenkie I, Dani R, Smith ER, et al. Ventricular interaction during experimental acute pulmonary embolism. Circulation 1988; 78 : 761-768.

37 Elzinga G, Piene $H$, de Jong JP. Left and right ventricular pump function and consequences of having two pumps in one heart. A study on the isolated cat heart. Circ Res 1980; 46: 564-574.

38 Overbeek MJ, Lankhaar JW, Westerhof N, et al. Right ventricular contractility in systemic sclerosis-associated and idiopathic pulmonary arterial hypertension. Eur Respir J 2008; 31: 1160-1166.

39 Kuehne T, Yilmaz S, Steendijk P, et al. Magnetic resonance imaging analysis of right ventricular pressure-volume loops: in vivo validation and clinical application in patients with pulmonary hypertension. Circulation 2004; 110: 2010-2016.

40 Kong D, Shu X, Pan C, et al. Evaluation of right ventricular regional volume and systolic function in patients with pulmonary arterial hypertension using three-dimensional echocardiography. Echocardiography 2012; 29: 706-712. 